This item was submitted to Loughborough's Research Repository by the author.

Items in Figshare are protected by copyright, with all rights reserved, unless otherwise indicated.

Spontaneous oscillations in a nonlinear delayed-feedback shunting model of the pupil light reflex

PLEASE CITE THE PUBLISHED VERSION

PUBLISHER

(C) American Physical Society

LICENCE

CC BY-NC-ND 4.0

REPOSITORY RECORD

Bressloff, P.C., and C.V. Wood. 2019. "Spontaneous Oscillations in a Nonlinear Delayed-feedback Shunting Model of the Pupil Light Reflex". figshare. https://hdl.handle.net/2134/1729. 


\title{
Spontaneous oscillations in a nonlinear delayed-feedback shunting model of the pupil light reflex
}

\author{
P. C. Bressloff and C. V. Wood \\ Nonlinear and Complex Systems Group, Department of Mathematical Sciences, Loughborough University, \\ Loughborough, Leicestershire LE11 3TU, United Kingdom
}

(Received 3 February 1998; revised manuscript received 29 April 1998)

\begin{abstract}
We analyze spontaneous oscillations in a second-order delayed-feedback shunting model of the pupil light reflex. This model describes in a simple fashion the nonlinear effects of both the iris and retinal parts of the reflex pathway. In the case of smooth negative feedback, linear stability analysis is used to determine the conditions for a Hopf bifurcation in the pupil area as a function of various neurophysiological parameters of the system such as the time delay and the strength of neural connections. We also investigate oscillation onset in the case of piecewise negative feedback and obtain an analytical expression for the period of oscillations. Finally, complex periodic behavior is shown to arise in the presence of mixed feedback.
\end{abstract}

[S1063-651X(98)05109-5]

PACS number(s): 87.10.+e, 42.66.-p

\section{INTRODUCTION}

The pupil light reflex is an important noninvasive tool for pinpointing certain types of disorder in visually impaired patients (see, e.g., [1], and references therein). Under normal operating conditions the reflex may be viewed as a nonlinear closed-loop feedback system for regulating the retinal light flux. A simple schematic diagram is shown in Fig. 1. The pupil area is determined by the interaction between constricting and dilating mechanisms. Pupil contraction is caused by excitation of the circular pupillary constriction muscle innervated by the parasympathetic fibers. The motor nucleus for this muscle is the Edinger-Westphal nucleus located in the midbrain. There are two main mechanisms for pupil dilation: (i) an active component arising from activation of radial pupillary dilator muscles along sympathetic fibers and (ii) a passive component involving inhibition of the EdingerWestphal nucleus. The sympathetic activity governing the pupil response is related mainly to the thalamus, hypothalamus, and reticular system and reflects emotional states, pain and attention.

One potential indicator of disease is an extension in the cycle period, termed pupil cycling time (PCT), of the rhythmic contraction and dilation of the pupil under "edge-light" conditions [2-4]. Under normal operating conditions the amount of light entering the eye is proportional to the pupil area [Fig. 2(a)]. In contrast to this, under edge-light conditions a narrow pencil of light (of constant illumination) is placed near the iris margin at a position denoted by a threshold $A_{\text {th }}$ so that some light enters the eye and the pupil contracts. The contraction of the pupil prevents the light beam from entering the eye and the pupil subsequently dilates. This allows the light to enter the eye again, the pupil contracts and a cycling process is thus initiated [see Fig. 2(b)]. There is a time delay $\tau$ of approximately $300 \mathrm{~ms}$ in the response of the pupil to changes in retinal illumination whereas the PCT can vary from 1-3 s. The range of values of the threshold $A_{\text {th }}$ for which the pupil oscillates is typically smaller than the total allowed physical variation of the pupil area, and varies from patient to patient, even in the absence of disease [4].
Another way of inducing spontaneous oscillations is to use an important experimental technique called clamping $[3,5,6]$. The feedback loop of the pupil light reflex is first "opened" by focusing a narrow beam of light on the center of the pupil in order to remove the shadowing effect of the iris on the retina [see Fig. 2(c)]. This is called a Maxwellian view. The retinal area exposed to the light beam is constant so that the total flux hitting the retina depends only on the light intensity and not the pupil area. The feedback loop is then reclosed electronically by altering the light intensity according to the measured pupil area. This experimental setup exploits the near synchrony of the pupillary activity of the two eyes. That is, when one eye is exposed to light, both eyes have an almost identical response, so that it is sufficient to measure the response of the unexposed eye. It can be shown that the clamped pupil light reflex exhibits oscillations along similar lines to edge-light conditions once the gain of the externally controlled feedback becomes sufficiently large $[3,5,6]$.

Recently, Longtin and Milton [7,8] developed a mathematical model of pupil cycling under edge-light or clamped conditions using a first-order nonlinear delay-differential equation. This described the dynamics of the pupil area in terms of a model of the iris muscle response to efferent activity. The latter was taken to be a logarithmic function of the retinal light flux, which is consistent with experimental data. Using a mixture of linear stability analysis and numeri-

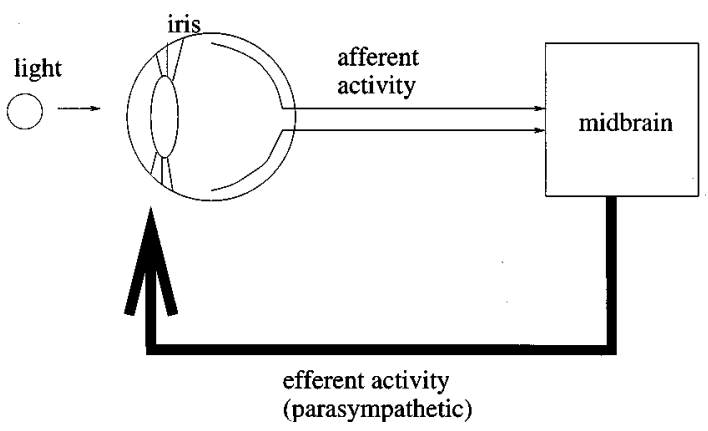

FIG. 1. Schematic diagram of the pupil light reflex showing negative feedback loop. 


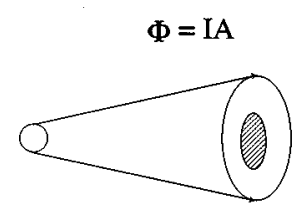

a) Closed-loop: normal

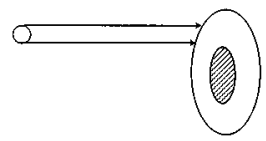

$\Phi=0$

$\mathrm{A}<\mathrm{A}_{\text {th }}$

b) Closed-loop: edge-light

FIG. 2. Operating configurations of the pupil light reflex. Here $I$ is the light intensity, $\Phi$ is total light flux entering pupil from given light source, $A$ is pupil area (shaded), $A_{r}$ is retinal illumination area in Maxwellian view, and $A_{\text {th }}$ is area threshold for edge light.

cal simulation, they showed that in the case of smooth feedback increasing the gain induced a supercritical Hopf bifurcation in the pupil area from a stable equilibrium point to a stable limit cycle. Although the Longtin-Milton model provides a good account of pupil cycling, it has a major limitation as a more general model of the pupil light reflex since it neglects any details concerning the dynamic response of the retina. As we have recently demonstrated, this results in an incorrect description of the pupil response to sinusoidally modulated light in an open-loop configuration [9]. Such behavior can be accounted for, however, by extending the Longtin-Milton model to include a phenomenological representation of the dynamics of the retinal system in the form of a leaky-integrator shunting equation [9]. The dynamics of the pupil light reflex is now described by a second-order delaydifferential equation, which can be decomposed into a pair of first-order delay-differential equations, one representing the iris component and the other the retinal component of the reflex arc.

In this paper, we study spontaneous oscillations in the extended Longtin-Milton model. The model is introduced in Sec. II where the response to forced oscillations under openloop conditions is described. We show how the results of Ref. [9] can be understood in terms of the concavity or convexity of the various nonlinearities present in the model. In Sec. III we use linear stability analysis to derive conditions for oscillation onset via a Hopf bifurcation in the case of smooth negative feedback. We show that the PCT near the bifurcation point lies in the range $(2 \tau, \infty)$ rather then $(2 \tau, 4 \tau)$ as in the original Longtin-Milton model. We then consider the case of piecewise constant negative feedback in Sec. IV. The latter, which corresponds to edge-light conditions, is not amenable to Hopf bifurcation analysis since the feedback is nondifferentiable. Instead, we extend the analysis of an der Heiden and Mackey [10] to derive an analytical expression for the pupil-cycling time under edge-light conditions. Finally, the complex dynamics associated with mixed feedback is studied in Sec. V. Importantly, we show that our model allows for closer agreement with experimental data on mixed feedback than the original Longtin-Milton model. Hence, the experimentally motivated second-order delay equation presented in this paper accounts for certain discrepancies of the previous first-order model both in open-loop and closed-loop configurations. Furthermore, it allows one to investigate the dependence of pupil light reflex dynamics on various neurophysiologically important parameters of the system such as the effective strength of neural connections and the time delay. These parameters vary from patient to patient and extreme values can be an indicator of a pathology.

\section{OPEN-LOOP RESPONSE TO FORCED OSCILLATIONS}

The Longtin-Milton model of the pupil light reflex represents the relation between the iris muscle activity and the retinal input in the form of a closed delay-differential equation for the pupil area $A(t)[7,8]$

$$
\frac{d g}{d A} \frac{d A}{d t}+\alpha g(A(t))=\gamma \ln \left[\frac{\Phi(t-\tau)}{\phi}\right], \quad \Phi(t)=I(t) A(t)
$$

with $I$ the light intensity, $\phi$ the threshold retinal illumination, and $\alpha$ and $\gamma$ positive constants. The time delay $\tau$ has contributions from the iris, neural pathways, and the retina. In Eq. (2.1) the efferent activity (parasympathetic) produced by the Edinger-Westphal nuclei is taken to be a logarithmic function of the retinal light flux. This incorporates the wellknown Weber-Fechner law [1]. The function $g$ is the inverse of the iris sphincter muscle function, $g=h^{-1}$, where $h$ relates muscle activity to pupil area, $A=h(x)$. An experimentally based choice for the function $h$ is the Hill function

$$
h(x)=\left(A_{\max }-A_{\min }\right) \frac{\Theta^{n}}{\Theta^{n}+x^{n}}+A_{\min }
$$

with $A_{\min }, A_{\max }$ the minimum and maximum pupil area, $\Theta$ is the value of $x$ at which the pupil area takes on its midrange value, and $n$ indicates the steepness of $h$ in midrange. Following Longtin and Milton [8] we take $A_{\max }=30 \mathrm{~mm}^{2}$, $A_{\min }=0 \mathrm{~mm}^{2}, \Theta=1 / 3$ (in dimensionless units), and $n=4$.

As mentioned in the Introduction, although the above model accounts reasonably well for spontaneous oscillations of the pupil light reflex under closed-loop conditions, it gives incorrect behavior in response to forced oscillations under open-loop conditions. Experimentally it is found that when the light-adapted pupil is exposed to sinusoidally modulated light without changing the average illumination, the pupil contracts further and the shift in average pupil size depends on the modulation frequency $\omega[11,4]$. The shift increases with $\omega$ at relatively low frequencies and decreases with $\omega$ at higher frequencies. The latter effect has a significant neural component, since it is observed at frequencies $(\geqslant 2 \mathrm{~Hz})$ above which the iris stops tracking the modulation. The smooth variation of the signal makes this behavior different from the contraction in response to sudden changes in light.

It is simple to show that the Longtin-Milton model actually generates a shift of the wrong sign, that is, it predicts that the pupil dilates rather than contracts. Moreover, this 
shift is frequency independent. Under open-loop conditions [Fig. 2(c)] $\Phi(t)$ in Eq. (2.1) becomes (for sinusoidally modulated light)

$$
\Phi(t)=I(t) A_{r}=\left\{\begin{array}{l}
I_{0} A_{r} \quad \text { if } t \leqslant 0 \\
\left(I_{0}+I_{1} \sin \omega t\right) A_{r} \quad \text { if } t>0
\end{array}\right.
$$

with $I_{1} / I_{0} \leqslant 1$ and $A_{r}$ is the constant illuminated retinal area. Suppose that the system is operating in its midrange, $A$ $\approx A^{*}=h(\Theta)$, so that $g$ can be approximated by a linear function

$$
g(A) \approx \beta\left(A-B^{*}\right), \quad \beta=g^{\prime}\left(A^{*}\right), \quad B^{*}=A^{*}-\beta^{-1} g\left(A^{*}\right),
$$

where $\beta<0$. Equation (2.1) can then be written in the form

$$
\begin{aligned}
\alpha^{-1} \frac{d A}{d t}+A(t) & =F(I(t)) \\
& \equiv \frac{\gamma}{\alpha \beta} \ln \left[\frac{\left[I_{0}+I_{1} \sin \omega(t-\tau)\right] A_{r}}{\phi}\right]+B^{*}
\end{aligned}
$$

(note that the delay has no dynamical effects in open loop). The equilibrium pupil area in the absence of periodic forcing is $A_{0}=F\left(I_{0}\right)$. For any periodic function $X(t)$ with period $T$, define the time average $\bar{X}$ by

$$
\bar{X}=\frac{1}{T} \int_{0}^{T} X(t) d t
$$

Ignoring transients, the average pupil area $\bar{A}$ in response to the given periodic forcing, [obtained by averaging Eq. (2.5) with respect to $t$ over a period $T]$, satisfies $\bar{A}=\overline{F(I)}$. The first point to note is that $\overline{F(I)}$ is frequency independent. Second, since $\ln$ is a concave function and $\gamma / \alpha \beta<0$, it follows that $\overline{F(I)} \geqslant F(\bar{I})=F\left(I_{0}\right)$. Therefore, $\bar{A}-A_{0} \geqslant 0$, that is, the model predicts incorrectly that the pupil dilates.

We shall now describe how to extend the above LongtinMilton model in order to obtain the correct response to periodic forcing [9]. The basic idea of the new model is to include a biologically plausible representation for the retinal component of the reflex arc, which incorporates the logarithmiclike compression of the retinal illumination $\Phi(t)$ $=I(t) A(t)$. This is modeled as a single unit by a standard leaky-integrator shunting equation leading to the system of equations

$$
\begin{gathered}
\frac{d V}{d t}=-\epsilon V(t)+[\Phi(t)-\phi][K-V(t)], \\
\frac{d g}{d A} \frac{d A}{d t}+\alpha g(A(t))=\gamma f(V(t-\tau)) .
\end{gathered}
$$

The additional dynamical variable $V$ is the membrane potential of the unit represented by the leaky integrator. The shunting effect describes the increase in synaptic conductance induced by the arrival of action potentials such that the change in the membrane potential $V$ depends on the difference between $V$ and a fixed membrane reversal potential $K$. The units of $V$ are fixed by taking $K=1$. In the leaky-integrator equation (2.7a) we are assuming that the change in synaptic conductance is proportional to the input light flux $\Phi(t)$ with the constant of proportionality set equal to unity for convenience. Such an equation is of course a gross simplification of the transduction process that converts incident light energy on the retina to nerve impulses emitted by retinal ganglion cells. The function $f$ represents the neural activation function. A biologically plausible choice for $f$ is the sigmoid function

$$
f(V)=\frac{1}{1+\exp \left[-\kappa\left(V-V_{\mathrm{th}}\right)\right]}
$$

with $\kappa, V_{\text {th }}$ constant. We shall set $\kappa=6 / K$ and $V_{\text {th }}=K$. We also take $\epsilon$ to be fixed in the range $10-100 \mathrm{~s}^{-1}$ (typical of a membrane decay rate) and $\alpha=3 \mathrm{~s}^{-1}$. Thus the only free parameters of our model will be the delay $\tau$, the strength of the efferent activity $\gamma$, and the specification of the light flux $\Phi(t)$.

We shall indicate how the additional equation (2.7a) can account for a number of important biological features in a simple manner. The inclusion of shunting implies that there exists an effective decay rate $\epsilon(t)$ for the membrane potential $V(t)$ that depends on the retinal flux $\Phi(t)$, that is,

$$
\epsilon(t)=\epsilon+\Phi(t)-\phi
$$

Equation (2.9) has several immediate consequences. First, it provides a mechanism for the experimentally observed asymmetry between fast pupil contraction and slow pupil dilation [1]. For increasing the retinal flux $\Phi(t)$ increases the effective decay rate and hence leads to a faster contraction than dilation. (Another contribution to this asymmetry is thought to arise from differences in the rate $\alpha$ of iris muscle contraction and dilation [7].) Second, shunting naturally gives a logarithmiclike compression of retinal response that is consistent with the Weber-Fechner law [12]. Indeed, in the presence of constant illumination $\Phi_{0}$ the steady-state response is

$$
V_{0}=\frac{K\left(\Phi_{0}-\phi\right)}{\epsilon+\Phi_{0}-\phi}
$$

Equation (2.10) shows that $V_{0} \rightarrow K$ as $\Phi_{0} \rightarrow \infty$ (saturation). The fact that $V$ saturates due to shunting implies that the maximum efferent activity is $\gamma f(K)$, which can be exploited as follows: (a) By choosing $V_{\mathrm{th}}=K$ we can restrict the system to operate in the convex domain of the sigmoid function $f$, that is, $f(V(t))<1 / 2$ for all $t$. This ensures that the model exhibits the correct open-loop response [9] (see below). (b) Equation (2.7b) shows that the maximum muscle activity is $x_{\max }=\gamma f(K) / \alpha$. Under normal operating conditions we would expect $h\left(x_{\max }\right) \approx A_{\min }$, that is, $x_{\max }=O(1)$. This immediately determines the scale of $\gamma$ to be $\gamma=O(\alpha)$.

Now suppose that $\Phi(t)$ is given by Eq. (2.3). Proceeding as in the analysis of the Longtin-Milton model by linearizing $g(A)$ according to Eq. (2.4) and averaging Eq. (2.7) over one period, we find that 


$$
\hat{\epsilon} \bar{V}=K\left(\Phi_{0}-\phi\right)-\Phi_{1} \hat{V}, \quad \bar{A}=\frac{\gamma}{\alpha \beta} \overline{f(V)}+B^{*}
$$

where $\hat{\epsilon}=\epsilon+\Phi_{0}-\phi, \Phi_{i}=I_{i} A_{r}, i=0,1$, and

$$
\hat{V}=\frac{1}{T} \int_{0}^{T} \sin (\omega t) V(t) d t .
$$

Suppose that the system is operating in the convex domain of the sigmoid function (2.8), which is reasonable for the lightadapted eye. Let $V_{0}, A_{0}$ be the equilibrium area and membrane potential in the absence of periodic forcing. Then we can write

$$
\bar{A}-A_{0}=\frac{\gamma}{\alpha \beta}\left\{[\overline{f(V)}-f(\bar{V})]+\left[f\left(V_{0}-\Phi_{1} \hat{V} / \hat{\epsilon}\right)-f\left(V_{0}\right)\right]\right\}
$$

It can be shown that $\hat{V}>0$ so that the second square bracket on the right-hand side of Eq. (2.13) is negative. On the other hand, the first square bracket is positive (convexity of $f$ ) and the net result is that the average shift $\delta A=\bar{A}-A_{0}<0$, that is, the pupil contracts. Moreover, the model predicts the correct frequency dependence for $\delta A$ above $2 \mathrm{~Hz}$. The low frequency behavior can also be accounted for by reintroducing the iris nonlinearity $g$. Thus all the nonlinearities present in the model contribute to the frequency-dependent shift in average pupil size when light is sinusoidally modulated.

Given the success of the extended Longtin-Milton model in accounting for the open-loop response to forced oscillations, we shall establish in the remaining sections that the model also exhibits the appropriate behavior in a closed-loop configuration, thus providing an interesting example of an experimentally motivated second-order delay-differential equation. Moreover, in the case of mixed negative feedback (Sec. V), we shall show that the second-order model accounts for certain discrepancies that the original first-order model has with experimental data.

\section{SMOOTH NEGATIVE FEEDBACK}

The system of delayed differential equations (2.7) under smooth negative feedback (clamped conditions) becomes

$$
\begin{gathered}
\frac{d V}{d t}=-\epsilon V(t)+\Phi(A(t), G)[K-V(t)], \\
\frac{d g}{d A} \frac{d A}{d t}+\alpha g(A(t))=\gamma f(V(t-\tau))
\end{gathered}
$$

with $\Phi(A(t), G)=I_{\text {ext }}(A(t), G) A_{r}$, where $A_{r}$ is the retinal area illuminated in Maxwellian view and $I_{\text {ext }}$ is the externally controlled light intensity, which depends both on the pupil area $A(t)$ and on some controllable gain parameter $G$. The total delay $\tau$ can also be manipulated by introducing an additional external delay in the reclosed feedback loop. For concreteness, we shall consider smooth negative feedback in which the light intensity is proportional to the pupil area [3]:

$$
\Phi(A, G)=G A \text {. }
$$

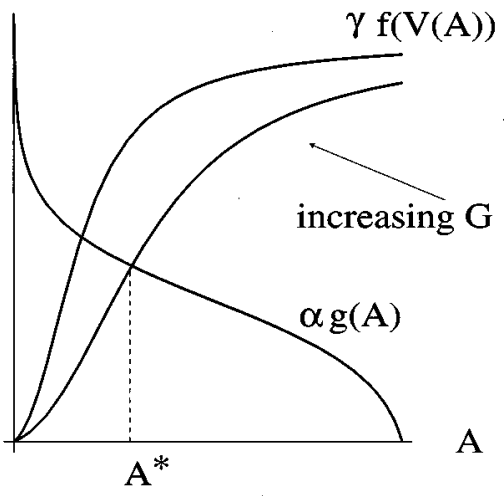

FIG. 3. Equilibrium area $A^{*}$ for smooth negative feedback with $V(A)$ defined by $V(A)=K G A /(\epsilon+G A)$.

The system (3.1) has one equilibrium point $\left(V^{*}, A^{*}\right)$ if the iris muscle function $g$ and the time constant $\alpha$ are considered the same for pupil dilation and contraction (see Fig. $3)$.

$$
\begin{gathered}
V^{*}=\frac{K \Phi\left(A^{*}, G\right)}{\epsilon+\Phi\left(A^{*}, G\right)} \\
\alpha g\left(A^{*}\right)=\gamma f\left(V^{*}\right) .
\end{gathered}
$$

We assume that $g^{\prime}(A) \neq 0$, for all $A$. Linearizing Eq. (3.1) about this equilibrium point gives

$$
\begin{gathered}
\frac{d v}{d t}=-\hat{\epsilon} v(t)+\mu a(t), \\
\frac{d a}{d t}=-\alpha a(t)+\eta v(t-\tau)
\end{gathered}
$$

with $v(t)=V(t)-V^{*}, a(t)=A(t)-A^{*}$, and

$$
\begin{gathered}
\hat{\boldsymbol{\epsilon}}=\boldsymbol{\epsilon}+\Phi\left(A^{*}, G\right), \\
\mu=\left.\left(K-V^{*}\right) \frac{\partial}{\partial A} \Phi(A, G)\right|_{A=A^{*}}, \\
\eta=\gamma \frac{f^{\prime}\left(V^{*}\right)}{g^{\prime}\left(A^{*}\right)}
\end{gathered}
$$

and $\hat{\epsilon}, \mu, \alpha>0, \eta<0$. The characteristic matrix $F(s)$ of the system is found by substituting $v(t)=v_{0} e^{s t}, a(t)=a_{0} e^{s t}$ into Eq. (3.5) to give

$$
F(s)\left(\begin{array}{c}
v_{0} \\
a_{0}
\end{array}\right)=0, \quad F(s)=\left(\begin{array}{cc}
s+\hat{\epsilon} & -\mu \\
-\eta e^{-\tau s} & s+\alpha
\end{array}\right) .
$$

The characteristic equation of the linear system is then $\operatorname{det} F(s)=0$, that is,

$$
s^{2}+(\hat{\epsilon}+\alpha) s+\alpha \hat{\epsilon}-\eta \mu \exp (-s \tau)=0 .
$$

The initial system of equations (3.1) is stable for $\tau=0$ and can undergo a change in stability of the equilibrium point when one or more roots of equation (3.10) for $\tau>0$ cross the imaginary axis. The characteristic equation (3.10) has no 

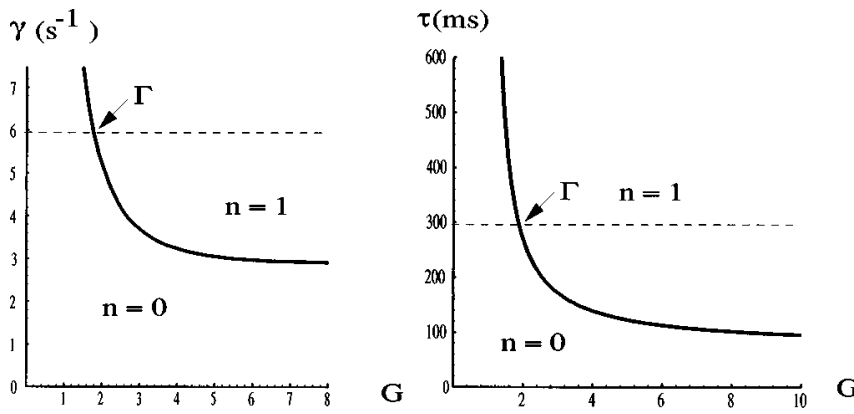

FIG. 4. (a) Stability curve in the plane $(G, \gamma)$ with $\tau=300 \mathrm{~ms}$. $\Gamma$ is the Hopf bifurcation point at $\gamma=6 \mathrm{~s}^{-1}$, with a change from stable fixed point to a stable limit cycle. (b) Stability curve for smooth negative feedback in the plane $(G, \tau)$ with $\gamma=6 \mathrm{~s}^{-1} . \Gamma$ is the Hopf bifurcation point at $\tau=300 \mathrm{~ms}$. In both diagrams $\alpha$ $=3 \mathrm{~s}^{-1}, \epsilon=50 \mathrm{~s}^{-1}, \Phi_{r}=20 \epsilon$, and the number of pairs of eigenvalues with positive real part is indicated by the value of $n$.

positive real roots $\lambda \geqslant 0$, since $s^{2}+(\hat{\boldsymbol{\epsilon}}+\alpha) s+\alpha \hat{\boldsymbol{\epsilon}}>0$ and $-\eta \mu \exp (-s \tau)>0$ for $s \geqslant 0$. Therefore, destabilization will involve at least one pair of complex roots crossing the imaginary axis. The conditions for the occurrence of a Hopf bifurcation can be determined from the following theorem due to Bellman and Cooke [13]:

Theorem: Let $H(z)=\left(z^{2}+p z+q\right) e^{z}+r$, where $p$ is real and positive, $q$ is real and non-negative, and $r$ is real. Denote by $a_{k}(k>0)$ the sole root of the equation $\cot a=\left(a^{2}-q\right) / p$, which lies on the interval $[(k-1) \pi, k \pi]$. We define the number $m$ as follows: (a) if $r \geqslant 0$ and $p^{2} \geqslant 2 q, m=1$; (b) if $r \geqslant 0$ and $p^{2}<2 q, m$ is the odd $k$ for which $a_{k}$ lies closest to $\sqrt{q-p^{2} / 2}$; (c) if $r<0$ and $p^{2} \geqslant 2 q, m=2$; (d) if $r<0$ and $p^{2}<2 q, m$ is the even $k$ for which $a_{k}$ lies closest to $\sqrt{q-p^{2} / 2}$.

Then a necessary and sufficient condition that all the roots of $H(z)=0$ lie to the left of the imaginary axis is that (i) $r$ $\geqslant 0$ and $\left(r \sin a_{m}\right) /\left(p a_{m}\right)<1$, or (ii) $-q<r<0$ and $\left(r \sin a_{m}\right) /\left(p a_{m}\right)<1$.

This theorem may be applied to Eq. (3.10) with $z=s \tau$, $p=\tau(\hat{\epsilon}+\alpha), q=\tau^{2} \hat{\epsilon} \alpha$, and $r=-\eta \mu \tau^{2}>0$. Thus the condition that all roots have a negative real part is condition (i) with $m=1$ [case (a)]. A pair of imaginary roots $\pm i \omega$ signaling the onset of a Hopf bifurcation occurs when $r \sin a_{1}$ $=p a_{1}$ with $a_{1}=\omega \tau$. From the definition of $a_{1}$ we deduce that $\omega$ is determined from the pair of equations

$$
\begin{aligned}
& \omega^{2}-\alpha \hat{\boldsymbol{\epsilon}}+\eta \mu \cos \omega \tau=0, \\
& (\hat{\boldsymbol{\epsilon}}+\alpha) \omega+\eta \mu \sin \omega \tau=0
\end{aligned}
$$

with $\omega \tau \in(0, \pi)$. Equations (3.11) and (3.12) can also be obtained by setting $s=i \omega$ in Eq. (3.10) and equating real and imaginary parts. We note that the period of oscillations $T$ $=2 \pi / \omega$ takes values in the range $(2 \tau, \infty)$ rather than $(2 \tau, 4 \tau)$ as in the Longtin-Milton model [8].

Figure 4(a) shows the stability curve for smooth negative feedback (3.2), which is obtained from numerical solutions of Eqs. (3.11) and (3.12) in the two dimensional subspace $(G, \gamma)$ with $\tau$ and $\Phi_{r}$ fixed. Note that $\eta, \mu, \hat{\epsilon}$, and $\omega$ all depend on $\gamma$ either directly or through the equilibrium point $\left(V^{*}, A^{*}\right)$. The region for which the equilibrium is stable (all

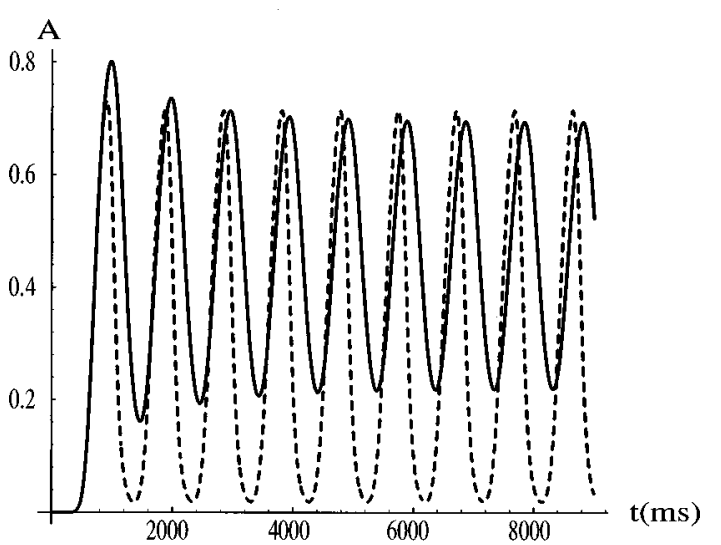

FIG. 5. Pupil oscillations for smooth negative feedback with $\alpha$ $=3 \mathrm{~s}^{-1}, \epsilon=50 \mathrm{~s}^{-1}, \tau=300 \mathrm{~ms}, \gamma=6 \mathrm{~s}^{-1}, \Phi_{r}=20 \epsilon$. Continuous line $G=1.9$, dashed line $G=5.0$. Pupil area is normalized with respect to $A_{\max }$.

eigenvalues have negative real part) is on the left-hand side of the stability curve. Here the conditions of the above theorem are satisfied. Provided that $\gamma$ is not too small, one finds that as one increases the gain $G$ the stability curve is crossed and the number of eigenvalues with positive real part changes from zero to two (point $\Gamma$ in Fig. 4). It turns out that the Hopf bifurcation is supercritical so that the point $\Gamma$ signals the onset of pupil cycling. For very small $\gamma$ one is below the stability curve and pupil cycling cannot occur for any $G$. These results are particularly interesting since the parameter $\gamma$ may be interpreted as a global neural connection strength so that a large reduction in $\gamma$ could signal some form of neurological damage. Similar considerations apply to the total delay time $\tau$ as illustrated in Fig. 4(b).

Analytical verification of the nondegeneracy conditions necessary for a Hopf bifurcation to be supercritical is quite involved for delay-differential equations, but it can be carried out systematically using a center manifold reduction as described in some detail by Campbell et al. [14,15] and Huang [16]. Following a similar analysis, one can establish that our model does indeed undergo a supercritical Hopf bifurcation [17]. The existence of a stable limit cycle can also be confirmed numerically by simulating the full model equations (3.1) as shown in Fig. 5 where we plot $A(t)$ for two different values of the gain $G$. We find that the amplitude of oscillations is an increasing function of $G$ except for large $G$. The period of oscillations is approximately constant, around $1 \mathrm{~s}$ for the parameter values $\alpha=3 \mathrm{~s}^{-1}, \epsilon=50 \mathrm{~s}^{-1}, \tau$ $=300 \mathrm{~ms}, \gamma=6 \mathrm{~s}^{-1}, \Phi_{r}=20 \epsilon$. These numerical results are consistent with the experimental data [3].

\section{PIECEWISE-CONSTANT NEGATIVE FEEDBACK}

In this section, we turn to the case of piecewise-constant negative feedback, which arises under edge-light conditions [Fig. 2(b)] or can be implemented using clamping and externally controlled feedback. Since the feedback is not described by a differentiable function, it is no longer possible to use techniques from Hopf bifurcation theory. Instead, we shall study the existence of periodic solutions along similar lines to an der Heiden and Mackey [10]. To find an analytical solution we consider a linearized iris function as in Eq. 
(2.4). The system of equations (3.1) becomes

$$
\begin{gathered}
\frac{d V}{d t}=-[\epsilon+\Phi(A(t))] V(t)+K \Phi(A(t)), \\
\frac{d A}{d t}=-\alpha A(t)+\frac{\gamma}{\beta} f(V(t-\tau))+\alpha B^{*}
\end{gathered}
$$

with

$$
\Phi(A)=\left\{\begin{array}{l}
0 \text { if } A \leqslant A_{\text {th }} \\
\Phi_{0} \text { if } A>A_{\text {th }}
\end{array}\right.
$$

and $A_{\text {th }}<A_{\text {max }}$. To simplify the analysis we shall assume that the synaptic input induced by the retinal light flux is large, $\Phi_{0} \gg \epsilon, \alpha$. Under this condition one can take $V(t) \approx K$ for all $t$ such that $A(t) \geqslant A_{\mathrm{th}}$. We consider the onset of edge-light stimulation at $t=t_{s}$ and for $t<t_{s}$ the variables $V(t), A_{\tau}(t)$, with $A_{\tau}(t)=A(t+\tau)$, are taken to be the steady-state solutions for constant light flux $\Phi_{0}$. That is, $V(t)=K, A_{\tau}(t)$ $=A_{\text {on }}$ for $t \leqslant t_{s}$, where

$$
A_{\mathrm{on}}=B^{*}+\frac{\gamma f(K)}{\alpha \beta} .
$$

This corresponds to an experimental setup in which the light source is moved from the center to the pupillary margin at the time $t=t_{s}$. We shall assume that $A_{\text {on }}<A_{\text {th }}$, otherwise $A_{\text {on }}$ would be a stable fixed point of the system. Finally, we linearize $f$ in Eq. (4.1b) by taking $f(V) \approx f(K)-(K$ $-V) f^{\prime}(K)$, and define

$$
A_{\text {off }}=B^{*}+\frac{\gamma}{\alpha \beta}\left[f(K)-K f^{\prime}(K)\right] .
$$

Let $t_{i}$, integer $i \geqslant 1$, denote the $i$ th time that the pupil area $A(t)$ crosses the threshold $A_{\text {th }}$, either from below (odd $i$ ) or from above (even $i$ ) and set $t_{0}=0$. Introduce the intervals $I_{i}=\left[t_{i-1}, t_{i}\right), i \geqslant 1$. If $t \in I_{i}$ for odd integer $i$ then $A(t)<A_{\text {th }}$ and $V(t)$ is an exponentially decreasing function of time, whereas, if $t \in I_{i}$ for even integer $i$ then $A(t) \geqslant A_{\text {th }}$ and $V(t)$ $=K$ (since $\Phi_{0}$ is large). Similarly, the time-shifted pupil area $A_{\tau}(t)$ is an increasing (decreasing) function of time for $t$ $\in I_{i}$ and $i$ an odd (even) integer. One can iteratively solve Eq. (4.1) for the pair $\left(V(t), A_{\tau}(t)\right)$ by integrating with respect to $t$ over each interval $I_{i}$ under the initial condition $\left(K, A_{\tau}\left(t_{i-1}\right)\right)$ with $A_{\tau}\left(t_{i-1}\right)$ the final state of the solution obtained over the previous interval $I_{i-1}$. It is useful to introduce the indexed set of functions

$$
\left(V^{i}(\theta), A_{\tau}^{i}(\theta)\right)=\left(V\left(t_{i-1}+\theta\right), A_{\tau}\left(t_{i-1}+\theta\right)\right),
$$

where $\theta \in\left[0, \Delta_{i}\right), \Delta_{i}=t_{i}-t_{i-1}$, and $i \geqslant 1$. Solving Eq. (4.1) then gives for $j \geqslant 1$

$$
\begin{gathered}
V^{2 j+1}(\theta)=K e^{-\epsilon \theta}, \\
A_{\tau}^{2 j+1}(\theta)=A_{\text {off }}+\left[A_{\tau}^{2 j+1}(0)-A_{\text {off }}+\frac{\alpha\left(A_{\text {off }}-A_{\text {on }}\right)}{\alpha-\epsilon}\right] e^{-\alpha \theta} \\
-\frac{\alpha\left(A_{\text {off }}-A_{\text {on }}\right)}{\alpha-\epsilon} e^{-\epsilon \theta}
\end{gathered}
$$

and

$$
\begin{gathered}
V^{2 j}(\theta)=K, \\
A_{\tau}^{2 j}(\theta)=A_{\text {on }}+\left[A_{\tau}^{2 j}(0)-A_{\text {on }}\right] e^{-\alpha \theta} .
\end{gathered}
$$

The solutions are patched together using the condition

$$
A_{\tau}^{i}\left(\Delta_{i}\right)=A_{\tau}^{i+1}(0) .
$$

We shall now prove that after an initial transient phase the system exhibits periodic behavior. We first note that $A_{\tau}^{2 j}\left(\Delta_{2 j}-\tau\right)=A_{\text {th }}$ for all $j \geqslant 1$. Equation (4.10) then implies that for all $j \geqslant 1$

$$
A_{\tau}^{2 j+1}(0) \equiv B=A_{\text {on }}+\left[A_{\text {th }}-A_{\text {on }}\right] e^{-\alpha \tau} .
$$

Thus $A_{\tau}^{2 j+1}(0)$ is independent of the initial data so that Eq. (4.7) has the same initial condition, and hence the same solution, for all $j \geqslant 1$. In particular $\Delta_{2 j+1}=\Delta_{d}$ for all $j \geqslant 1$ where $\Delta_{d}$ is the solution to the equation $A_{\mathrm{th}}=A_{\tau}^{2 j+1}\left(\Delta_{d}\right.$ $-\tau$ ), which yields

$$
\begin{aligned}
A_{\mathrm{th}}-A_{\text {off }}= & {\left[B-A_{\text {off }}+\frac{\alpha\left(A_{\text {off }}-A_{\text {on }}\right)}{\alpha-\epsilon}\right] e^{-\alpha\left(\Delta_{d}-\tau\right)} } \\
& -\frac{\alpha\left(A_{\text {off }}-A_{\text {on }}\right)}{\alpha-\epsilon} e^{-\epsilon\left(\Delta_{d}-\tau\right)} .
\end{aligned}
$$

It also follows that $A_{\tau}^{2 j-1}\left(\Delta_{d}\right)$ is independent of $j$ for all $j$ $\geqslant 2$ so that from Eqs. (4.7) and (4.10)

$$
\begin{aligned}
A_{\tau}^{2 j}(0) \equiv \bar{B}= & A_{\text {off }}+\left[B-A_{\text {off }}+\frac{\alpha\left(A_{\text {off }}-A_{\text {on }}\right)}{\alpha-\epsilon}\right] e^{-\alpha \Delta_{d}} \\
& -\frac{\alpha\left(A_{\text {off }}-A_{\text {on }}\right)}{\alpha-\epsilon} e^{-\epsilon \Delta_{d}}
\end{aligned}
$$

Equation (4.13) implies that Eq. (4.9) has the same initial condition $\bar{B}$, and hence the same solution, for all $j \geqslant 2$. In particular, $\Delta_{2 j}=\Delta_{c}$ for all $j \geqslant 2$ with $\Delta_{c}$ the solution to the equation $A_{\mathrm{th}}=A_{\tau}^{2 j}\left(\Delta_{c}-\tau\right)$, which can be solved explicitly to give

$$
\Delta_{c}=\frac{1}{\alpha} \ln \left[\frac{\bar{B}-A_{\text {on }}}{A_{\mathrm{th}}-A_{\mathrm{on}}}\right]+\tau .
$$

We conclude from the above analysis that Eq. (4.1) has a periodic solution $\left(V(t), A_{\tau}(t)\right)$ for all $t>t_{2}=\Delta_{1}+\Delta_{2}$ and the pupil cycling time is

$$
T=\Delta_{c}+\Delta_{d}
$$

with $\Delta_{c}$ and $\Delta_{d}$ determined by Eqs. (4.14) and (4.12), respectively. The amplitude of the oscillation is given by $\bar{B}$ $-B$. A numerical example of an oscillatory solution in the case of large $\Phi_{0}$ is shown in Fig. 6. A phase portrait of limit cycle oscillations in the space $\left(A_{\text {th }}, A(t-\tau), A(t)\right)$ for various values of the threshold $A_{\text {th }}$ is plotted in Fig. 7.

The feedback system described by Eqs. (4.1) and (4.2) also exhibits oscillatory behavior for smaller values of $\Phi_{0}$, except that now the solution is obtained asymptotically in the 

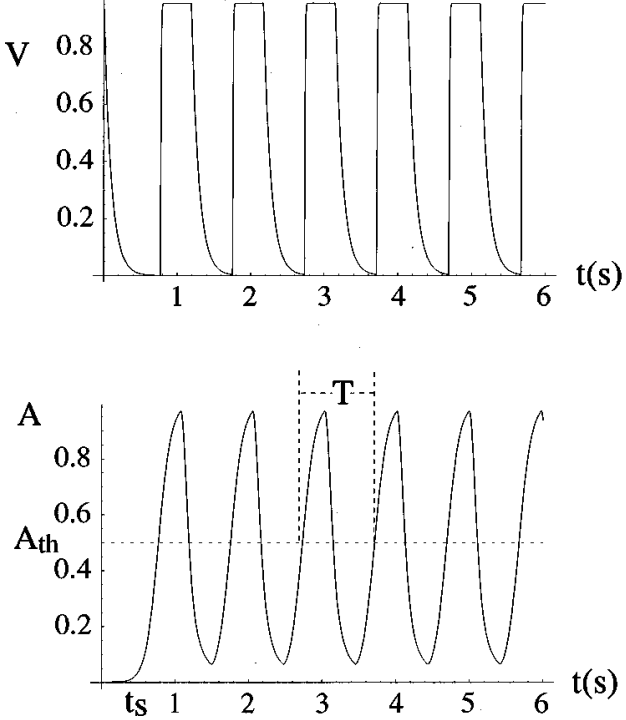

FIG. 6. Pupil oscillations for piecewise linear negative feedback with $A_{\mathrm{th}} / A_{\max }=0.5$ and $t_{s}$ the time for onset of "edge-light" stimulation. Here $\alpha=3 \mathrm{~s}^{-1}, \epsilon=10 \mathrm{~s}^{-1}, \tau=300 \mathrm{~ms}, \gamma=6 \mathrm{~s}^{-1}$, and $\Phi_{r}$ $=20 \epsilon$. The variables $A$ and $V$ are normalized with respect to $A_{\max }$ and $K$, respectively.

limit $t \rightarrow \infty$ rather than after a finite interval. In practice, this convergence is very rapid, as can be seen in Fig. 8. We shall briefly indicate how to determine the resulting periodic solution. Following the previous analysis of the strong flux case, Eqs. (4.5)-(4.9), we can decompose the periodic solution into a contracting phase and a dilating phase:

$$
\lim _{j \rightarrow \infty}\left(V^{2 j}(\theta), A_{\tau}^{2 j}(\theta)\right)=\left(V^{c}(\theta), A^{c}(\theta)\right), \quad \theta \in\left[0, \Delta_{c}\right),
$$

$$
\lim _{j \rightarrow \infty}\left(V^{2 j+1}(\theta), A_{\tau}^{2 j+1}(\theta)\right)=\left(V^{d}(\theta), A^{d}(\theta)\right), \quad \theta \in\left[0, \Delta_{d}\right],
$$

where $T=\Delta_{c}+\Delta_{d}$ is the period of oscillation. We also have the initial conditions

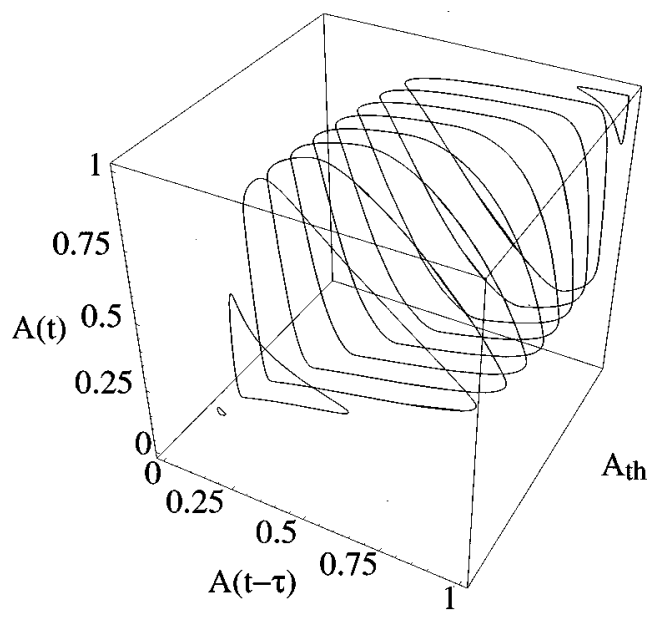

FIG. 7. Phase plane for pupil cycling with the parameter pupil area $A_{\text {th }}$ as a third dimension. Parameters are as in Fig. 6.
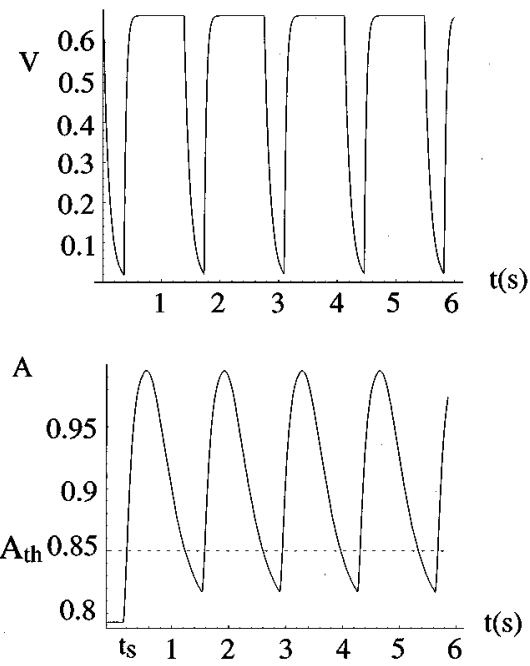

FIG. 8. Pupil oscillations for piecewise linear negative feedback for small light flux. All parameter values are as in Fig. 6 except that $A_{\text {th }} / A_{\text {max }}=0.85$ and $\Phi_{r}=2 \epsilon$.

$$
\begin{array}{ll}
V^{c}(0) & =U, \quad V^{d}(0)=\bar{U}, \\
A^{c}(0) & =\bar{B}, \quad A^{d}(0)=B,
\end{array}
$$

where $U$ and $\bar{U}$ are the minimum and maximum values of the periodic solution for $V(t)$. Similarly, $B$ and $\bar{B}$ are the minimum and maximum values of the periodic solution for $A_{\tau}(t)$. The functions $V^{c, d}(\theta)$ are found by solving Eqs. (4.1a) and (4.18) to give

$$
V^{c}(\theta)=U e^{-\hat{\epsilon} \theta}+\frac{K \Phi_{0}}{\hat{\epsilon}}\left[1-e^{-\hat{\epsilon} \theta}\right], \quad V^{d}(\theta)=\bar{U} e^{-\epsilon \theta},
$$

where $\hat{\epsilon}=\Phi_{0}+\epsilon$. Similarly, the functions $A_{\tau}^{c, d}(\theta)$ are obtained by solving Eqs. (4.1b) and (4.19) using the solutions for $V_{c, d}(\theta)$. The result is

$$
\begin{aligned}
& A^{c}(\theta)=A_{\mathrm{on}}+\Lambda^{c} e^{-\alpha \theta}+\Gamma^{c} e^{-\hat{\epsilon} \theta}, \\
& A^{d}(\theta)=A_{\mathrm{off}}+\Lambda^{d} e^{-\alpha \theta}+\Gamma^{d} e^{-\epsilon \theta},
\end{aligned}
$$

where

$$
\begin{gathered}
A_{\mathrm{on}}=B^{*}+f_{0}+\frac{K \Phi_{0} f_{1}}{\hat{\epsilon}}, \quad \Lambda^{c}=\bar{B}-A_{\mathrm{on}}-\Gamma^{c}, \\
\Gamma^{c}=\frac{\alpha f_{1}}{\alpha-\hat{\epsilon}}\left[U-\frac{K \Phi_{0}}{\hat{\epsilon}}\right], \\
A_{\mathrm{off}}=B^{*}+f_{0}, \quad \Lambda^{d}=B-A_{\mathrm{off}}-\Gamma^{d}, \quad \Gamma^{d}=\frac{\alpha f_{1}}{\alpha-\epsilon} \bar{U} .
\end{gathered}
$$

In deriving these equations, we have linearized $f(V)$ in Eq. (4.1b) about a mid-range point $V^{*}<K$ and set $\gamma f(V) /(\alpha \beta)$ $\approx f_{0}+f_{1} V$. Finally, the unknown amplitudes $U, \bar{U}, B$, and $\bar{B}$ together with the periods $\Delta_{c, d}$ are determined self- 
consistently from the following conditions [obtained by matching up the contracting and dilating solutions in Eqs. (4.20) and (4.21)]:

$$
\begin{gathered}
V^{d}\left(\Delta_{d}\right)=V^{c}(0), \quad V^{c}\left(\Delta_{c}\right)=V^{d}(0), \\
A^{c}\left(\Delta_{c}\right)=A^{d}(0), \quad A^{d}\left(\Delta_{d}\right)=A^{c}(0), \\
A^{c}\left(\Delta_{c}-\tau\right)=A_{\mathrm{th}}, \quad A^{d}\left(\Delta_{d}-\tau\right)=A_{\mathrm{th}} .
\end{gathered}
$$

\section{MIXED FEEDBACK}

It is instructive to make more explicit the connection between Eqs. (4.1) and (4.2) and the class of piecewiseconstant delayed-feedback models considered by an der Heiden and Mackey [10]. Let $a(t)=A(t)-A_{\text {on }}$ and $a_{\text {th }}$ $=A_{\text {th }}-A_{\text {on }}$ and assume that $\epsilon$ is sufficiently large so that we can take $V(t)=K$ whenever $a(t)>a_{\mathrm{th}}$ and $V(t)=0$ whenever $a(t) \leqslant a_{\mathrm{th}}$. Equations (4.1) and (4.2) then reduce to a single delay equation for the pupil area:

$$
\frac{d a}{d t}=-\alpha a(t)+h(a(t-\tau)),
$$

where

$$
h(a)=\left\{\begin{array}{l}
\alpha\left(A_{\mathrm{off}}-A_{\mathrm{on}}\right) \quad \text { if } a \leqslant a_{\mathrm{th}} \\
0 \quad \text { if } a>a_{\mathrm{th}}
\end{array} .\right.
$$

Equations (5.1) and (5.2) correspond to the type of negative feedback model analyzed in Ref. [10] and is found in the original Longtin-Milton model.

For many physiological control systems an equation of the form (5.1) arises with $h[a(t-\tau)]$ given by a humped function of $a(t-\tau)$ for which maximal input occurs at some intermediate value of $a(t-\tau)$. In other words, the control system displays both positive and negative feedback characteristics. Analytical and numerical studies have demonstrated that mixed feedback systems can exhibit a complex variety of periodic and aperiodic (chaotic) dynamics [10]. Of particular interest here concerns the experimental investigation of the dynamical effects of mixed feedback in the pupil light reflex $[18,19]$. This was carried out under clamped conditions with piecewise-constant feedback of the form

$$
\Phi(A)=\left\{\begin{array}{l}
0 \text { for } A \leqslant A_{1} \text { and } A \geqslant A_{2} \\
\Phi_{0} \text { for } A_{1}<A<A_{2} .
\end{array}\right.
$$

The lower threshold $A_{1}$ and the higher threshold $A_{2}$ were externally controlled. Equation (5.2) then becomes

$$
h(a)=\left\{\begin{array}{l}
\alpha\left(A_{\mathrm{off}}-A_{\mathrm{on}}\right) \quad \text { for } a_{1}<a<a_{2} \\
0 \quad \text { for } a \leqslant a_{1} \text { and } a \geqslant a_{2} .
\end{array}\right.
$$

Interestingly, a discrepancy between the prediction of the original first-order Longtin-Milton model and the experimentally observed oscillations of the pupil under mixed feedback was found; the model predicted too many changes in pupil area per cycle in a certain parameter regime [see Fig. 3(e) of Ref. [19] and Fig. 7(c) of Ref. [18]]. This raises the issue of whether or not the second-order model can account for such a discrepancy, and hence give an improvement upon the (a)

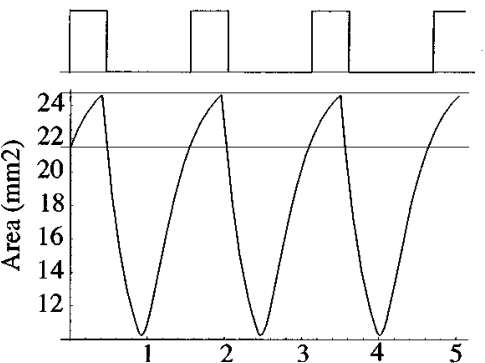

(b)
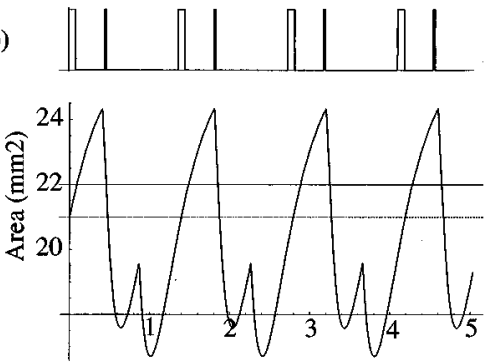

(c)
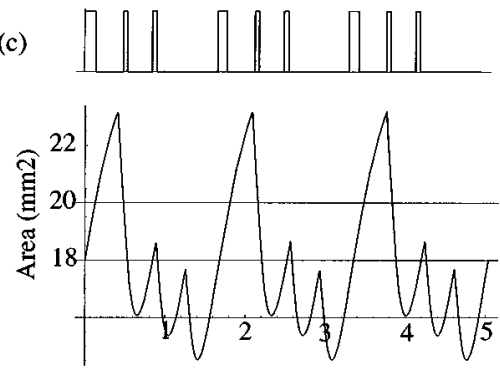

Time (sec)

FIG. 9. Pupil oscillations for mixed feedback and various choices of thresholds $A_{1}$ and $A_{2}$. Here $\epsilon=6 \mathrm{~s}^{-1}, \alpha=3 \mathrm{~s}^{-1}, \gamma$ $=6 \mathrm{~s}^{-1}, \quad \tau=411 \mathrm{~ms}, \quad \Phi_{r}=20 \epsilon$. (a) $A_{1}=21.5 \mathrm{~mm}^{2}, A_{2}$ $=24.75 \mathrm{~mm}^{2}$, (b) $A_{1}=21 \mathrm{~mm}^{2}, A_{2}=22 \mathrm{~mm}^{2}$, (c) $A_{1}=18 \mathrm{~mm}^{2}$, $A_{2}=20 \mathrm{~mm}^{2}$.

original model with respect to closed-loop as well as openloop response. It turns out that this is indeed the case, as is illustrated in Fig. 9. As the two thresholds $A_{1}$ and $A_{2}$ are varied, the system undergoes changes in the number of pulses of light received by the pupil, which is very suggestive of the data presented in the cited figures. Such behavior is also found to be quite robust. A more detailed investigation of mixed feedback will be presented elsewhere [20].

\section{DISCUSSION}

In this paper, we have presented a mathematical model of the pupil light reflex in terms of a system of nonlinear delaydifferential equations. This model incorporates experimentally determined physiological parameters that underlie the complex dynamical behavior of the pupil light reflex, and nonlinear techniques such as Hopf bifurcation theory have been used to study the onset of this behavior. The model extends the Longtin-Milton model $[7,8]$ by including a phenomenological representation of the dynamics of the retinal system in the form of a leaky integrator shunting equation. The resulting flux-dependent modulation of the effective time constant of the system (due to shunting), combined with the various nonlinearities of the system, accounts for a wide range of observed features: (i) the asymmetry between pupil 
contraction and dilation (ii) the frequency-dependent shift in the average pupil response when a constant light stimulus is modulated by a sinusoidal input, (iii) the nonlinear summation of signals from left and right eyes (binocular versus monocular sinusoidal response), and (iv) spontaneous oscillations under conditions of high gain negative feedback. Future work will investigate the effects of noise arising from the neural components of the reflex arc, as well as details concerning photoreceptor dynamics. In particular, the important role that photoreceptors play in light adaptation will be investigated and contrasted with possible neural mechanisms for adaptation. The latter can be modeled as a stochastic process, with neuronal units becoming desynchronized due to differences in their firing patterns after an initial synchronized burst caused by illumination onset.

In conclusion, the pupil light reflex is an important paradigm for nonlinear feedback control systems. Understanding the behavior of such systems involves important mathematical questions concerning the properties of differential equations with delays and noise, and could benefit the clinician interested in developing diagnostic tests for detecting neurological disorders. It is also hoped that the work will have applications in other areas such as respiratory and cardiac control.
[1] I. E. Loewenfeld, The Pupil: Anatomy, Physiology, and Clinical Applications (Iowa State University Press, Detroit, 1993).

[2] S. D. Miller and H. S. Thompson, Brit. J. Ophthalmol. 62, 495 (1978).

[3] J. G. Milton, A. Longtin, T. H. Kirkham, and G. S. Francis, Am. J. Ophthalmol. 105, 402 (1988).

[4] P. A. Howarth, G. Heron, and L. Wittaker (unpublished).

[5] L. Stark, J. Opt. Soc. Am. 52, 925 (1962).

[6] J. G. Milton and A. Longtin, Vision Res. 30, 515 (1990).

[7] A. Longtin and J. G. Milton, Phys. Rev. A 41, 6992 (1990).

[8] A. Longtin, J. G. Milton, J. E. Bos, and M. C. Mackey, Bull. Math. Biol. 51, 602 (1989).

[9] P. C. Bressloff, C. V. Wood, and P. A. Howarth, Proc. R. Soc. London, Ser. B 263, 953 (1996).

[10] U. an der Heiden and M. C. Mackey, J. Math. Biol. 6, 75 (1982).

[11] D. Varjú, in Processing of Optical data by Organisms and
Machines, edited by W. Reichardt (Academic Press, New York, 1969), pp. 442-464.

[12] S. Grossberg, Neural Networks 1, 17 (1988).

[13] R. Bellman and K. L. Cooke, Differential-Difference Equations (Academic Press, London, 1963).

[14] S. A. Campbell, J. Bélair, T. Ohira, and J. G. Milton, Chaos 5, 640 (1995).

[15] S. A. Campbell, J. Bélair, T. Ohira, and J. G. Milton, J. Dyn. Diff. Eqns. 7, 213 (1995).

[16] Q. Huang and J. Wei, Lecture Notes in Pure and Applied Math 176, 107 (1996).

[17] C. V. Wood, Ph.D. thesis, Loughborough University, 1998.

[18] A. Longtin and J. G. Milton, Math. Biosci. 90, 183 (1988).

[19] J. G. Milton, A. Longtin, A. Beuter, M. C. Mackey, and L. Glass, J. Theor. Biol. 138, 129 (1989).

[20] P. C. Bressloff and C. V. Wood (unpublished). 